\title{
Real-Time Disparity Contrast Combination for Onboard Estimation of the Visibility Distance
}

\author{
Nicolas Hautière, Raphaël Labayrade, and Didier Aubert
}

\begin{abstract}
An atmospheric visibility measurement system capable of quantifying the most common operating range of onboard exteroceptive sensors is a key parameter in the creation of driving assistance systems. This information is then utilized to adapt sensor operations and processing or to alert the driver that his onboard assistance system is momentarily inoperative. Moreover, a system capable of either detecting the presence of fog or estimating visibility distances constitutes in itself a driving assistance. In this paper, the authors present a technique to estimate the mobilized visibility distance through a use of onboard charge-coupled device cameras. The latter represents the distance to the most distant object on the road surface having a contrast above 5\%. This definition is very close to the definition of the meteorological visibility distance proposed by the International Commission on Illumination. The method combines the computations of local contrasts above $5 \%$ and of a depth map of the vehicle environment using stereovision within $60 \mathrm{~ms}$ on a current-day computer. In this paper, both methods are described separately. Then, their combination is detailed. The method is operative night and day in every kind of meteorological condition and is evaluated; thanks to video sequences under sunny weather and foggy weather.
\end{abstract}

Index Terms-Charge coupled devices camera, contrast impairment, driving assistance, fog, meteorological visibility, stereovision.

\section{INTRODUCTION}

$\mathbf{P}$ ERCEPTION sensors (cameras, laser, radar, etc.) are being introduced into certain vehicles. These sensors have been designed to operate within a wide range of situations and conditions (weather, luminosity, etc.) with a prescribed set of variation thresholds. Effectively detecting when a given operating threshold has been surpassed constitutes a key parameter in the creation of driving assistance systems that meet the required reliability levels. With this context in mind, an atmospheric visibility measurement system may be capable of quantifying the most common operating range of onboard exteroceptive sensors. This information is then utilized to adapt sensor operations and processing, to automate tasks such as turning on fog lamps or alert the driver that his onboard assistance system is momentarily inoperative. Moreover, a system capable of either detecting the presence of fog or estimating visibility distances constitutes, in itself, driving assistance. Indeed, during foggy

Manuscript received June 14, 2005; revised October 3, 2005. This work was supported in part by the French ARCOS project. The Associate Editor for this paper was H. Takahashi.

The authors are with the Vehicles-Infrastructure-Drivers Interactions Research Laboratory (LIVIC), a joint French National Institute For Transport and Safety Research-French Public Works Research Laboratory (INRETS-LCPC) entity, 78000 Versailles Satory, France (e-mail: nicolas.hautiere@lcpc.fr; raphael.labayrade@1cpc.fr; didier.aubert@inrets.fr).

Digital Object Identifier 10.1109/TITS.2006.874682 weather, humans actually tend to overestimate visibility distances [1], which can lead to excessive driving speeds.

Koschmieder's law [2] models the fog effects on the atmospheric visibility. One of its parameters is the extinction coefficient $k$ of fog. This parameter is strongly related to the meteorological visibility distance suggested by the International Commission on Illumination (CIE). Thus, we developed a technique estimating $k$ [3]. However, in order to cover more situations, that is, solely daytime foggy weather, we have developed a more generic approach, which is the topic of this paper. Thus, we estimate the greatest distance at which a picture element on the road surface is visible. We called it the mobilized visibility distance. We will see that this concept is close to the meteorological visibility. Then, to estimate this distance, we must carry out two tasks. First, we have to compute contrasts higher than a given threshold in the image. Then, we need to compute a depth map of the vehicle environment. Finally, we have to combine both.

\section{Current State of Knowledge}

In this section, we present Koschmieder's model, on which our work is based. Then, we describe quickly what exists in the literature.

\section{A. Koschmieder's Model}

In 1924, Koschmieder [2] proposed his theory on the apparent luminance of objects observed against background sky on the horizon. In noting that a distant object winds up blending in with the sky, he established a simple relationship between the distance $d$ of an object with intrinsic luminance $L_{\mathrm{o}}$ and its apparent luminance $L$ as follows:

$$
L=L_{\mathrm{o}} e^{-k d}+L_{f}\left(1-e^{-k d}\right)
$$

where $L_{f}$ denotes the luminance of the sky, and $k$ the extinction coefficient of the atmosphere.

Based on these results, Duntley [2] derived an attenuation law of atmospheric contrasts

$$
C=C_{\mathrm{o}} e^{-k d}
$$

where $C$ designates the apparent contrast at distance $d$ and $C_{\mathrm{o}}$ the intrinsic contrast of the object against its background.

This law is only applicable in the case of uniform illumination of the atmosphere. In order for the object to be just barely visible, the value of $C$ must equal the contrast threshold $\varepsilon$. From a practical standpoint, the CIE [2] has adopted an average value 
of $\varepsilon=0.05$ for the contrast threshold so as to define a conventional distance called the "meteorological visibility distance" $V_{\text {met }}$, i.e., the greatest distance at which a black object $\left(C_{\mathrm{o}}=1\right)$ of a suitable dimension can be seen in the sky on the horizon

$$
V_{\text {met }}=-\frac{1}{k} \ln (0.05) \simeq \frac{3}{k} .
$$

\section{B. Visibility Distance Estimation Through a Use of a Camera}

The use of a camera to estimate the visibility distance has only received minimal attention in literature. Most relevant approaches employ a camera fastened to the road structure, which simplifies the measurement operation given that a reference image is always available. Bush and Debes [4] relied upon a fixed camera placed above the roadway for the purpose of measuring visibility distances.

Systems that entail a use of an onboard camera, however, are encountered much less frequently. Pomerleau [5] estimated visibility by means of measuring a contrast attenuation per meter on the road markings at various distances in front of a moving vehicle. Instead of estimating a contrast attenuation per meter, he could have estimated the meteorological visibility distance. Owing to (1), we know $L_{m}$ (road luminance) and $L_{M}$ (road marking luminance) variations according to the distance to the camera. By taking two distances $d_{1}$ and $d_{2}, k$ could be expressed as follows:

$$
k=\frac{1}{d_{2}-d_{1}} \ln \left(\frac{L_{M_{1}}-L_{m_{1}}}{L_{M_{2}}-L_{m_{2}}}\right) .
$$

Finally, we could obtain the meteorological visibility distance

$$
V_{\mathrm{met}}=3 \frac{d_{2}-d_{1}}{\ln \left(\frac{L_{M_{1}}-L_{m_{1}}}{L_{M_{2}}-L_{m_{2}}}\right)}
$$

However, this approach, based on the "RALPH" system [5], requires the presence and detection of road markings in order to proceed.

Yahiaoui and Da Silva Dias [6] estimate the quality of images for the human eye by comparing the modulation transfer function of current images to the contrast sensibility function from Mannos and Sakrison [7] but only returns a potential visibility distance.

In [3], Hautière and Aubert succeed to instantiate Koschmieder's model and then to estimate the meteorological visibility distance. This method, when its operation assumptions are met, lead to good results under daytime foggy weather.

\section{Generic Method Proposal}

\section{A. Mobilized Visibility Distance}

For the CIE, the meteorological visibility distance is the greatest distance at which a black object of a suitable dimension can be seen in the sky on the horizon. We have decided to build a method that is close to this definition. In this aim, we propose to study the distance to the most distant object having enough contrast with respect to its background.

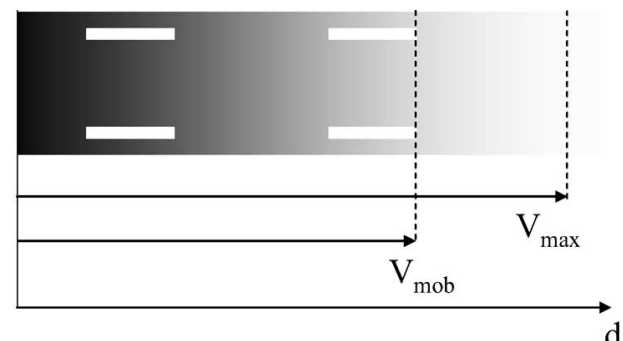

(a)

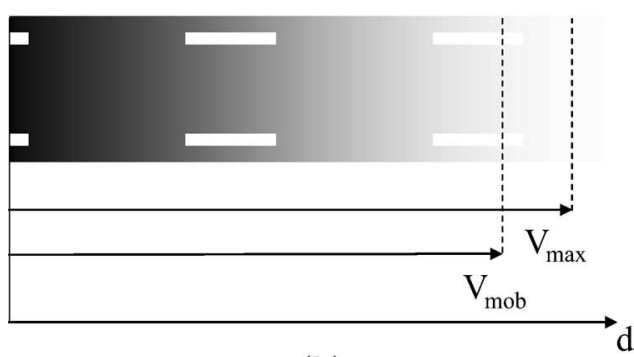

(b)

Fig. 1. Examples of mobilized and mobilizable visibility distances. The mobilized visibility distance $V_{\mathrm{mob}}$ is the distance to the most distant visible object existing on the road surface. The mobilizable visibility distance $V_{\max }$ is the greatest distance at which a potential object on the road surface would be visible.

In Fig. 1, we represent a simplified road with dash road marking. In Fig. 1(a), we suppose that the most distant visible object is the extremity of the last road marking (it could have been the border of the road too). In Fig. 1(b), the vehicle has moved and a new road marking is now visible. We call this distance to the most distant visible object, which depends on the road scene, the mobilized visibility distance $V_{\text {mob }}$. This distance has to be compared to the mobilizable visibility distance $V_{\max }$. This is the greatest distance at which a picture element on the road surface would be visible.

Consequently, we have the following relationship:

$$
V_{\max } \geq V_{\text {mob }}
$$

\section{B. Mobilizable Visibility Distance}

In this section, we are going to establish the link between the mobilizable visibility distance and the meteorological visibility distance. The mobilized visibility distance is the distance to the most distant object $W$ considered as visible. We denote $L_{b_{\mathrm{o}}}$ and $L_{w_{\mathrm{o}}}$ the intrinsic luminances and $L_{b}$ and $L_{w}$ the luminances at the distance $d$ of the road $B$ and the object $W$.

Koschmieder's law gives us the theoretical variations of these values according to the distance $d$. Let us express the contrast $C_{B W}$ of $W$ with respect to $B$ like Weber does (15):

$$
C_{B W}=\frac{\Delta L}{L}=\frac{\left(L_{w_{\mathrm{o}}}-L_{b_{\mathrm{o}}}\right) e^{-k d}}{L_{b_{\mathrm{o}}} e^{-k d}+L_{f}\left(1-e^{-k d}\right)} .
$$

We deduce the expression of $d$ according to the photometric parameters, the contrast $C_{B W}$, and the fog density $k$ :

$$
d=-\frac{1}{k} \ln \left(\frac{C_{B W} L_{f}}{L_{w_{\mathrm{o}}}-L_{b_{\mathrm{o}}}+C_{B W}\left(L_{f}-L_{b_{\mathrm{o}}}\right)}\right) .
$$


That is to say the distance where an object $W$ is perceived with a contrast of $C_{B W}$. Owing to (3), we can express this value according to the meteorological visibility distance $V_{\text {met }}$

$$
d=-\frac{V_{\text {met }}}{3} \ln \left(\frac{C_{B W} L_{f}}{L_{w_{\mathrm{o}}}-L_{b_{\mathrm{o}}}+C_{B W}\left(L_{f}-L_{b_{\mathrm{o}}}\right)}\right) .
$$

Like CIE does, we can choose a threshold $\tilde{C}_{B W}$ below where the object is considered as being not visible. Like for the computation of the meteorological visibility distance, we assume that the road intrinsic luminance is equal to zero. Then, we define the mobilizable visibility distance $V_{\max }$ valid for every threshold contrast

$$
V_{\max }=\max _{\left.\left.L_{w_{\mathrm{o}}} \in\right] 0, M\right]}-\frac{V_{\mathrm{met}}}{3} \ln \left(\frac{\tilde{C}_{B W} L_{f}}{L_{w_{\mathrm{o}}}+\tilde{C}_{B W} L_{f}}\right) .
$$

The energy received by the object $W$ is not entirely reflected toward the camera. Consequently, we have the following relationship:

$$
L_{w_{\mathrm{o}}} \leq L_{f} .
$$

We deduce the value of $V_{\max }$

$$
V_{\max }=-\frac{V_{\mathrm{met}}}{3} \ln \left(\frac{\tilde{C}_{B W}}{1+\tilde{C}_{B W}}\right) .
$$

Then, we easily obtain the value $\tilde{C}_{B W}$ so that $V_{\max }=V_{\text {met }}$

$$
\tilde{C}_{B W}=\frac{1}{e^{3}-1} \approx 5 \% \text {. }
$$

Therefore, by choosing a contrast threshold $\tilde{C}_{B W}$ of $5 \%$, the mobilizable visibility distance is close to the meteorological visibility distance $V_{\text {met }}$ for a black object.

Actually, the road is never black and the sky rarely white. The mobilizable visibility distance represents a maximum of visibility distance rarely reachable, since it is the greatest distance at which the clearest object is visible on a black road. On the other hand, the mobilized visibility distance, which only takes into account the gray objects encountered in the image, is the distance that we are able to estimate directly. This distance is precisely the one we want to estimate in this paper.

\section{Proposed Method}

In Section III-A, we have introduced the concepts of mobilized and mobilizable visibility distances. Whereas the first one depends on the road scene, the second one only depends on the meteorological conditions. Then, in Section III-B, we established the link between the meteorological visibility distance defined by the CIE and the mobilizable visibility distance previously defined. In particular, we calculated the contrast threshold so that both distances are the same, that is, to say $5 \%$. Consequently, we propose to estimate the mobilized visibility distance by estimating the distance to the most distant object on the road surface having a contrast above $5 \%$. This method is decomposed in two tasks. The first one consists of computing the contrasts in the image and selecting the ones above 5\%. The second one is the depth computation of the detected picture elements and the selection of the most distant one.

\section{Computation of Local Contrasts Above 5\%}

The method to develop has to be accurate, since it must only detect contrasts above or equal to 5\%. Then, it has to go fast because the application must be performed in real time on a moving vehicle, but also be robust to the noise present in the image. Finally, it has to be adapted to the contrast definition used by the CIE to define the meteorological visibility distance.

Moreover, our work is based on the assumption that the conversion process between incident energy on the chargecoupled device (CCD) sensor and the grey level value in the image is linear, which is generally the case for short exposure times. In fact, we use short exposure times on our onboard cameras, so as to reduce the motion blur. Consequently, our assumption can be considered as valid.

In this part, we present first the literature on contrast measurement. Then, we focus on our technique and explain why it is well fitted to our objectives.

\section{A. Related Work}

Different definitions of the contrast exist. One of the most famous is Michelson's contrast [7]. It has been introduced to quantify the visibility of sinusoidal gratings

$$
C^{M}=\frac{L_{\max }-L_{\min }}{L_{\max }+L_{\min }} .
$$

where $L_{\min }$ and $L_{\max }$ are the minimal and maximum luminance values of the image. The use of sinusoidal gratings and of this contrast definition has met a great success in psychophysics. In particular, it has been used to study the human eye by building contrast sensitivity functions (CSF).

Weber [7] defined the contrast as being a relative luminance variation $\Delta L$ with respect to the background $L$. This has been used to measure the visibility of targets

$$
C^{W}=\frac{\Delta L}{L} .
$$

This contrast definition is sometimes called psychophysical contrast and is used in the definition of the meteorological visibility distance.

These definitions are good estimators of contrast for the stimuli previously mentioned: sinusoïdal gratings for Michelson, uniform targets for Weber. However, they are not well adapted when the stimulus becomes more complex. Moreover, none of these definitions are adapted to estimate the contrast in natural images. This is mainly due to the fact that the contrast perception is local. This is these local methods on which we focused our attention.

In the image quality assessment field, other contrast definitions exist [7], [8]. Many of them try to model the human vision by a contrast sensitivity function. However, in this case, spatial frequency of the encountered objects in the image has 

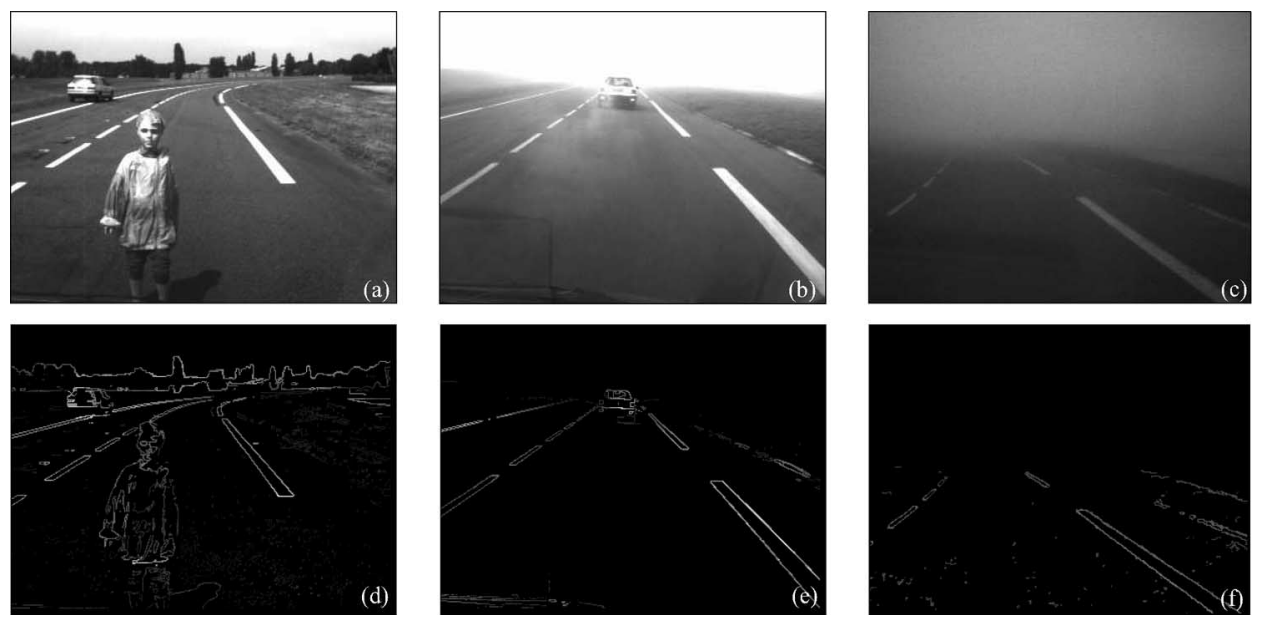

Fig. 2. Images captured in the vehicle (a) under sunny weather, (b) under foggy weather, and (c) under dense foggy weather before nightfall. Examples of computation of contrasts above $5 \%$ on the whole images (d) for image (a), (e) for image (b), and (f) for image (c).

to be known as well as their depth. Without hypothesis on the scene structure, like a flat world (cf. [6]), such a modeling is hazardous.

In their logarithmic image processing framework, Jourlin and Pinoli [9] defined the logarithmic contrast available in transmitted light between two pixels $x$ and $x_{1}$ of an image $f$. They are among the first to define the concept of local contrast

$$
C_{x, x_{1}}(f)=\frac{M\left|f(x)-f\left(x_{1}\right)\right|}{M-\min \left(f(x), f\left(x_{1}\right)\right)}
$$

where $M$ is the maximum gray value in the considered scale.

Gordon's method [10] also defines the concept of local contrast. This method computes Michelson's contrast between the mean value of two concentric regions. Beghdadi [8] proposed a method inspired from Gordon's method. It takes into account the mean value of the edges detected in the considered location.

On the opposite, methods that restore image contrast under adverse weather conditions are much more encountered in literature. Unfortunately, they have all of them strong constraints and, consequently, they cannot be installed onboard a moving vehicle. Some techniques require prior information about the scene (constant altitude) [11], while others require dedicated hardware in order to estimate the weather conditions (diffusiometer, transmitiometer...). Some techniques rely only upon the acquired images and exploit the atmospheric scattering to obtain the range map of the scene [12], [13]. However, they require weather conditions to change between image acquisitions. Narasimhan and Nayar [12] proposed such an impressive method. Otherwise, polarization filter techniques can be used to reduce haziness in the image. Unfortunately, they require two differently filtered images of the same scene. This is the case for Schechner [14] who analyzed two filtered images taken in adverse weather to compute scene structure and dehaze them.

\section{B. Measuring the Contrast With Köhler's Thresholding Technique}

We propose an original method, inspired from Köhler's technique, and we show why it is suitable to our situation.
1) Principle: Köhler's technique [15] finds the threshold that maximizes the contrast between two parts of the image. Let $f$ be a gray level image. A couple of pixels $\left(x, x_{1}\right)$ is said to be separated by the threshold $s$ if two conditions are met. First, $x_{1} \in V_{4}(x)$. Second, we have

$$
\min \left(f(x), f\left(x_{1}\right)\right) \leq s<\max \left(f(x), f\left(x_{1}\right)\right) .
$$

Let $F(s)$ be the set of all couples $\left(x, x_{1}\right)$ separated by $s$, such as $x \in V_{4}\left(x_{1}\right)$. With these definitions, for every value of $s$ belonging to [0,255[, $F(s)$ is built. For every couple belonging to $F(s)$, the contrast $C_{x, x_{1}}(s)$ is calculated as

$$
C_{x, x_{1}}(s)=\min \left(|s-f(x)|,\left|s-f\left(x_{1}\right)\right|\right) .
$$

The mean contrast (19) associated to $F(s)$ is then performed

$$
C(s)=\frac{1}{\operatorname{card}(F(s))} \sum_{\left(x, x_{1}\right) \in F(s)} C_{x, x_{1}}(s) .
$$

The best threshold $s_{\mathrm{O}}$ respects the following condition:

$$
C\left(s_{\mathrm{o}}\right)=\max _{s \in[0,255[} C(s)
$$

where $s_{\mathrm{O}}$ is the threshold that has the best mean contrast along the associated border $F\left(s_{\mathrm{o}}\right)$. Instead of using this method to binarize images, we use it to measure the contrast locally. The evaluated contrast is equal to $2 C\left(s_{\mathrm{o}}\right)$ along the associated border $F\left(s_{\mathrm{o}}\right)$. Examples of contrast computations are shown in Fig. 2.

2) Adaptation to the Logarithmic Contrast: The previous method is suitable for different definitions of local contrast. We only need to use the adequate definition in the place of (18). In our case, we have chosen to estimate the logarithmic contrast [9], which is also used by the CIE to define the meteorological visibility distance. Thus, (18) becomes

$$
C_{x, x_{1}}(s)=\min \left(\frac{|s-f(x)|}{\max (s, f(x))}, \frac{\left|s-f\left(x_{1}\right)\right|}{\max \left(s, f\left(x_{1}\right)\right)}\right) .
$$


(a)

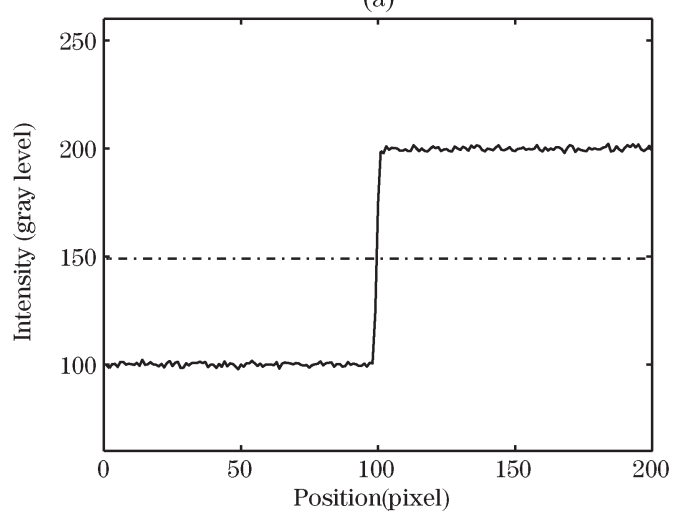

(c)

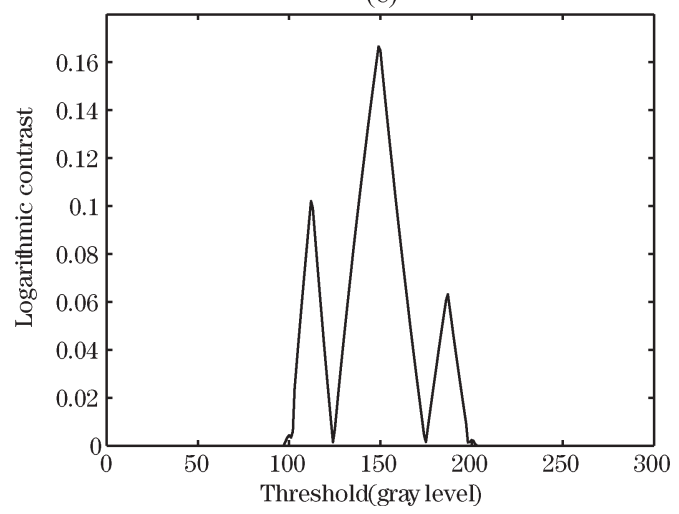

(b)

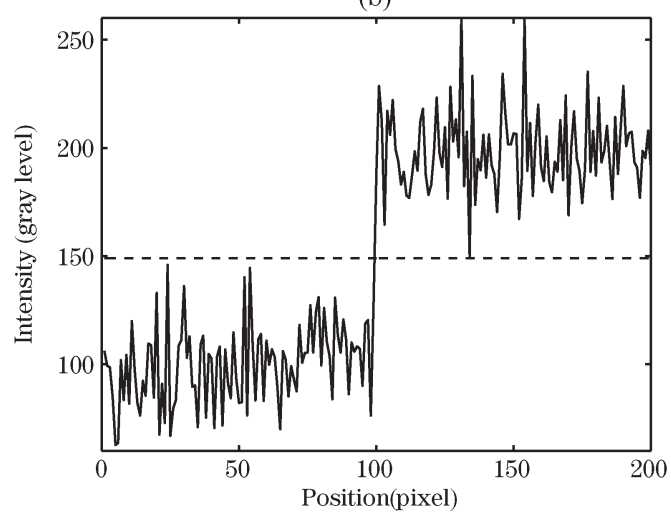

(d)

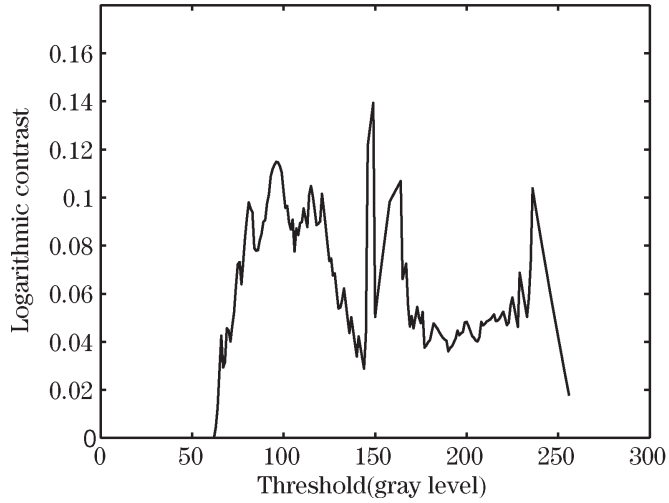

Fig. 3. Noise robustness of Köhler's method adapted to the logarithmic contrast. One-dimensional edge modified by Gaussian noise (a) $\sigma=1$ and (b) $\sigma=17$. The dotted line represents the optimal threshold found by the method. The mean contrast $C(s)$ associated with each threshold value $s$ is plotted for (c) $\sigma=1$ and (d) $\sigma=17$.

For the Michelson contrast, $C_{x, x_{1}}$ would be expressed in the following way:

$$
C_{x, x_{1}}(s)=\min \left(\frac{|s-f(x)|}{s+f(x)}, \frac{\left|s-f\left(x_{1}\right)\right|}{s+f\left(x_{1}\right)}\right) .
$$

3) Noise Robustness: The method derived from Köhler is robust to noise. We assume that the noise of the camera is Gaussian. This assumption is confirmed in Section VI-B. Let us consider two Gaussian distributions of means $L_{1}$ and $L_{2}$ and standard deviations $\sigma_{1}$ and $\sigma_{2}$. We can show that, as long as both distributions are not intersected, the optimal threshold $s_{\mathrm{O}}$ found by Köhler's technique is a Gaussian distribution with mean $\left(L_{1}+L_{2}\right) / 2$ and standard deviation $(1 / 2) \sqrt{\sigma_{1}^{2}+\sigma_{2}^{2}}$.

Consequently, the method is robust to noise, because in average, the returned threshold is the one without noise at the same distance of both distributions. This property is still verified when using the local formula of logarithmic contrast. Fig. 3 illustrates this property.

Fig. 3(a) and (b) has the same distributions with additive Gaussian noise of standard deviation $\sigma=1$ and $\sigma=17$, respectively. The optimal threshold found by Köhler's technique, which is represented by the horizontal dashed line, is the same for both distributions. It is the one that gives the maximum contrast [cf. Fig. 3(c) and (d)].

On the opposite, if both distributions are intersected, i.e., if $\max \left(3 \sigma_{1}, 3 \sigma_{2}\right)>\left(L_{2}-L_{1}\right) / 2$, Köhler's technique is no longer so efficient.
4) Algorithm Improvements: From an algorithmic point of view, the technique is rather expensive, in particular, the computation of the border $F(s)$ for each threshold of the gray scale. A first improvement consists in decreasing the number of thresholds considered by seeking the minimal and maximum intensities. To compute $F(s)$, the scanning of the computation window is made from top to bottom and from left to the right. Thus, to consider only the vicinity $V_{4}^{*}$ (cf. Fig. 4) makes it possible to take into account each couple of items only once, reducing the computing time. We can also consider the vicinity $V_{8}^{*}$. However, the tests carried out show that the difference is tiny between the approaches $V_{4}$ and $V_{8}$. Taking into account the saving of computing time in approach $V_{4}$, we thus use the vicinity $V_{4}^{*}$ to carry out the scanning process. The last major improvement consists in computing the images $I_{\max }^{g}, I_{\min }^{g}$, $I_{\max }^{h}$, and $I_{\min }^{h}$ before scanning the image $I$

$$
\begin{aligned}
I_{\max }^{g} & =\left\{p \in I / p=\max \left(p, p_{g}\right)\right\} \\
I_{\min }^{g} & =\left\{p \in I / p=\min \left(p, p_{g}\right)\right\} \\
I_{\max }^{h} & =\left\{p \in I / p=\max \left(p, p_{h}\right)\right\} \\
I_{\min }^{h} & =\left\{p \in I / p=\min \left(p, p_{h}\right)\right\}
\end{aligned}
$$

where $p_{g}$ is the pixel on the left and $p_{h}$ the pixel above the current pixel.

Thereafter, instead of computing the minimum and maximum to build the border, it is enough to look at the adequate image. In this way, the method is much faster to carry out. 
(a)

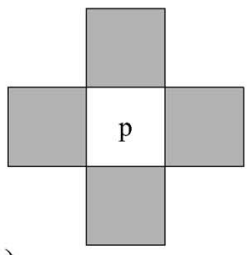

(b)

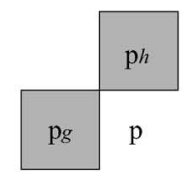

(c)
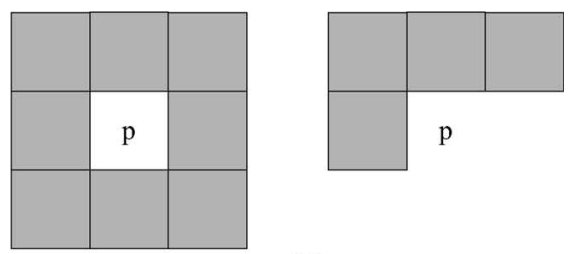

(d)

Fig. 4. Different vicinities of a pixel. (a) $V_{4}$, (b) $V_{4}^{*}$, (c) $V_{8}$, and (d) $V_{8}^{*}$. In particular, $V_{4}^{*}$ is the one we have chosen to compute the min-max images.

TABLE I

Computation Time of Contrasts Above 5\% According to Fourmodes : Without Optimization, With Minimization of the Number of Thresholds Considered, With Precalculation of the Min-Max Images, and With Both Preceding Optimizations. The Ratio Between the Computing Time Without and With in C Language

\begin{tabular}{|c|c|c|c|c|}
\hline & \multicolumn{4}{|c|}{$\begin{array}{c}\text { Computing time in seconds } \\
\text { (ratio original method/optimized method) }\end{array}$} \\
\hline $\begin{array}{c}\text { Vicinity size } \\
\text { (pixel) }\end{array}$ & $\begin{array}{c}\text { Direct } \\
\text { computation }\end{array}$ & $\begin{array}{c}\text { Minimization of } \\
\text { threshold number }\end{array}$ & $\begin{array}{c}\text { Precalculation } \\
\text { MIN-MAX images }\end{array}$ & $\begin{array}{c}\text { Minimization }+ \\
\text { Precalculation }\end{array}$ \\
\hline 2 & $14,1(1)$ & $2,2(6,4)$ & $3,4(4,1)$ & $1(14,1)$ \\
8 & $10,3(1)$ & $2,6(4)$ & $2(5,1)$ & $0,83(12,4)$ \\
16 & $8,3(1)$ & $3(2,75)$ & $1,7(4,9)$ & $0,81(10,2)$ \\
\hline
\end{tabular}

If one wishes to use a vicinity $V_{8}^{*}$, there are as many images to precompute.

Table I shows the interest of algorithmic optimizations previously described. The computing time of contrasts above $5 \%$ is given according to fourmodes: without optimization, with minimization of the number of thresholds considered, with precalculation of the min-max images, and with both preceding optimizations. The ratio between the computing time without and with optimization is given between brackets. The saving of computation time is higher than ten times for the vicinity sizes usually considered. The tests are carried out on an Intel Pentium IV $2.4 \mathrm{GHz}$ in $\mathrm{C}$ language without specific compilation. By using compiler Intel C++ 8.0, the computing time is less than $350 \mathrm{~ms}$.

\section{Robust Estimation of Picture-Element Depth}

\section{A. Background}

If just a single camera is used, we are unable to gain access to the image depth. This problem can be overcome by adopting the hypothesis of a flat world, which makes it possible to associate a distance with each line of the image. However, the depth on vertical objects is incorrect and is unknown without another assumption. In a first approach, we can detect picture elements belonging to the road surface. Techniques that search the road surface are numerous. A first family of methods finds the road surface by a segmentation process. Color segmentation [16] and texture segmentation [17] are the main approaches. A second family of methods finds the road surface by detection of its edges [18]-[20].

Conversely, we can detect the objects above the road surface. Some techniques are based on optic flow computation [21]. However, it is time consuming, and the main hypothesis is not always verified (spatio-temporal luminous flow preserved). Some methods rely on template matching [22] or local symmetry [23] but are necessarily not generic.
In addition, techniques like depth from scattering [12], depth from focus/defocus [24], and depth from shading [25] are not adapted to our objectives.

If we use stereovision, we are not limited to the flat world hypothesis, and we are able to gain access to the depth of nearly every pixels in the image [26]. However, because of real-time constraints, most approaches compute a sparse disparity map. Our approach belongs to this family, because we only need depth information where contrast is above $5 \%$, that is, to say on the edges. We present our approach in the next section.

\section{B. "v-Disparity" Approach}

1) Image of a Plane in the " $v$-Disparity" Image: The stereovision algorithm uses the "v-disparity" transform, in which the detection of straight lines is equivalent to the detection of planes in the scene. In this aim, we represent the $v$ coordinate of a pixel toward the disparity $\Delta$ (performing accumulation from the disparity map along scanning lines) and detect straight lines and curves in this "v-disparity" image (denoted by $I_{v_{\Delta}}$ ) [27].

This algorithm assumes the road scene is composed of set of planes: obstacles are modelized as vertical planes, whereas the road is supposed to be a horizontal plane (when it is planar), or a set of oblique planes (when it is not planar), as shown in Fig. 5.

According to the modeling of the stereo sensor given in Fig. 6, the plane of equation $Z=d$, corresponding to a vertical object, is projected along the straight line of (23) in $I_{v_{\Delta}}$

$$
\Delta=\frac{b}{d}\left(v-v_{\mathrm{o}}\right) \sin \theta+\frac{b}{d} \alpha \cos \theta
$$

The plane of equation $Y=0$, corresponding to the road surface, is projected along the straight line of (24) in $I_{v_{\Delta}}$

$$
\Delta=\frac{b}{h}\left(v-v_{\mathrm{o}}\right) \cos \theta+\frac{b}{h} \alpha \sin \theta
$$




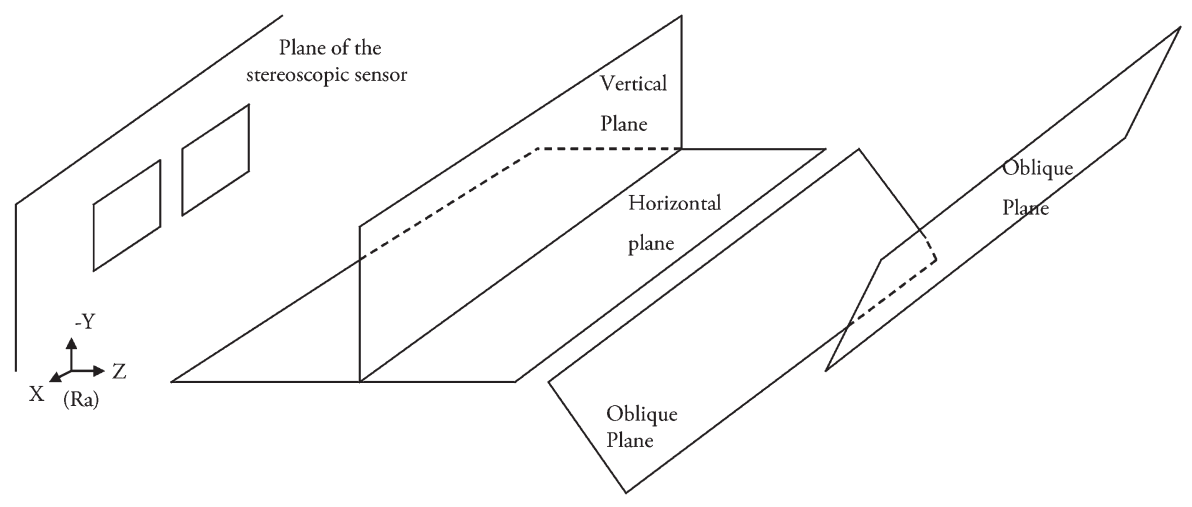

Fig. 5. Domain of the validity of the study.

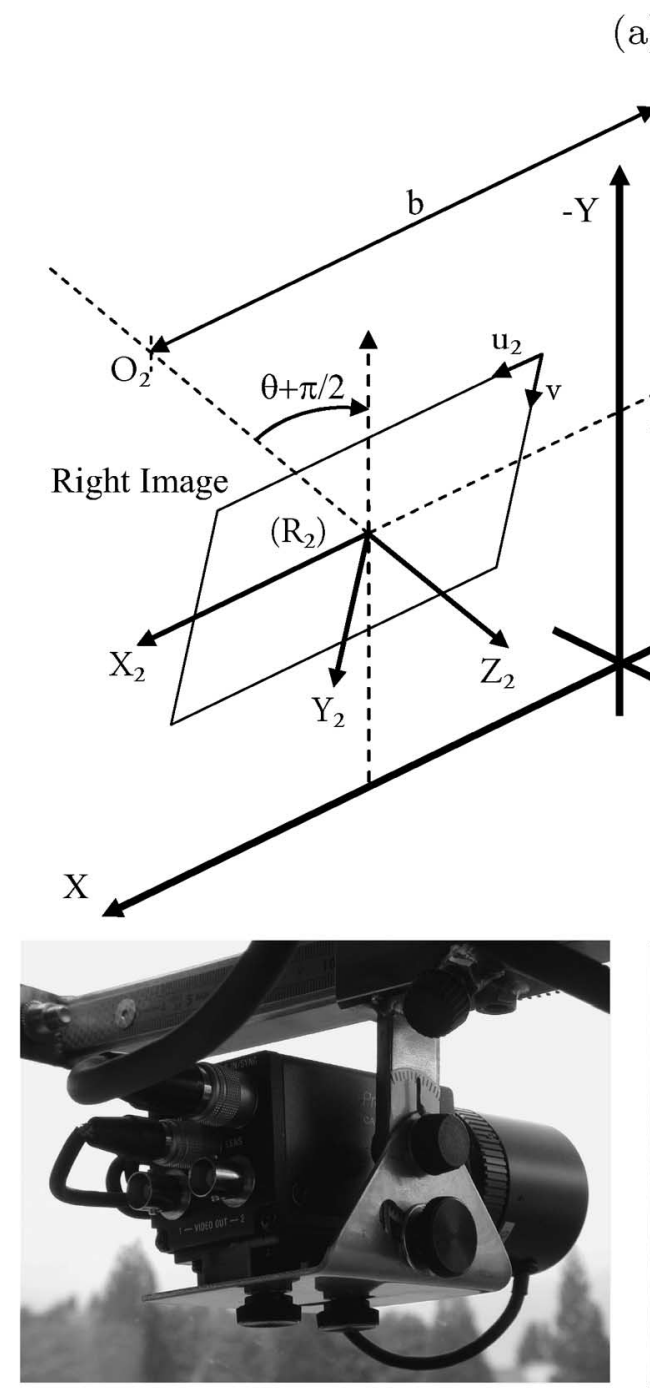

(b)

(a)
$\mathrm{O}_{1} \quad$ Left image $\mathrm{u}_{1}$

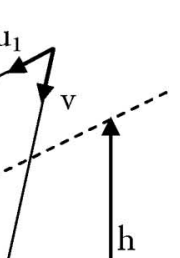




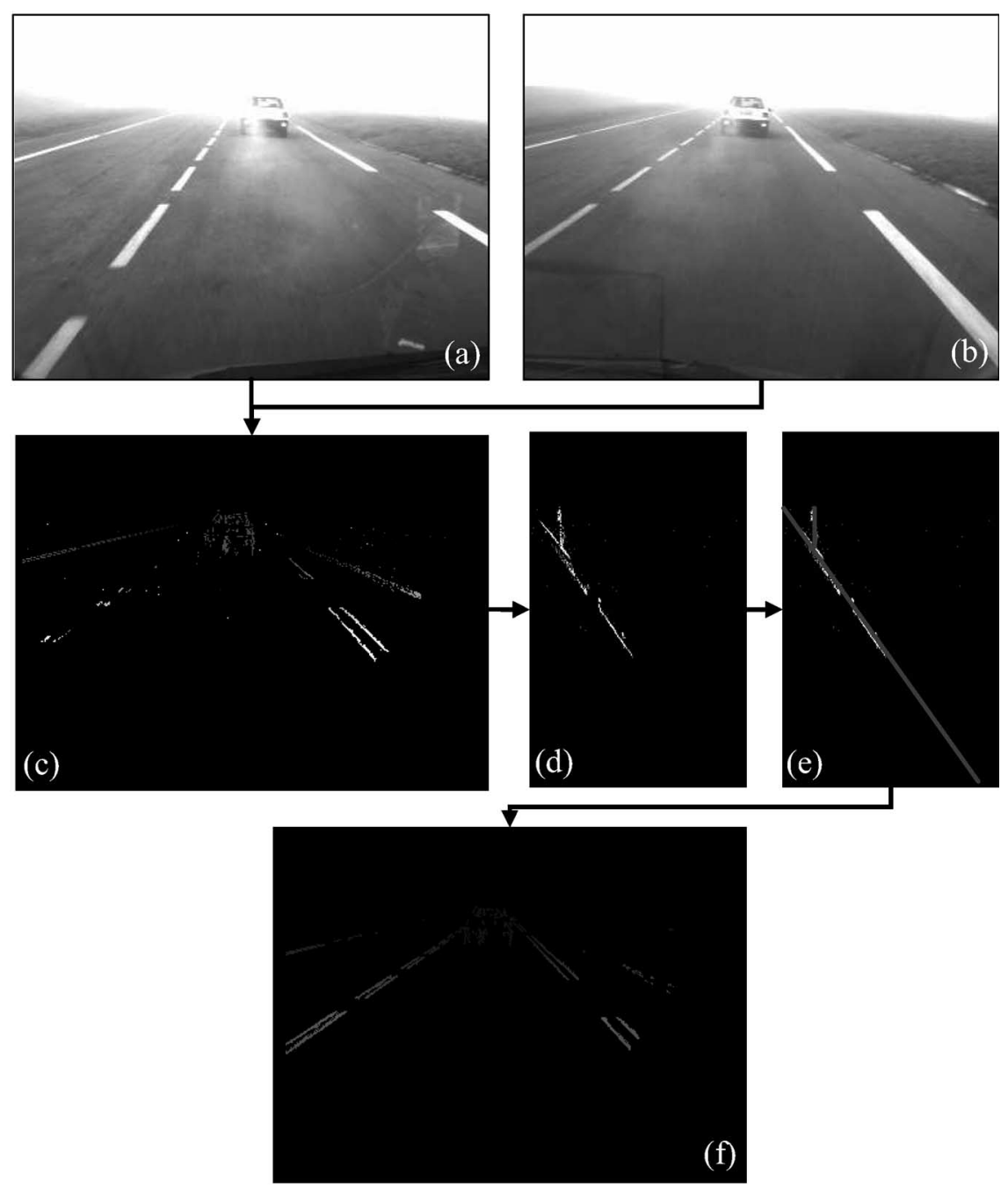

Fig. 7. Overview of the "v-disparity" framework. (a) Left original image; (b) right original image; (c) rough disparity map computed from images (a) and (b); (d) "v-disparity" image; (e) extracted lines from the "v-disparity" image; and (f) improved disparity map.

performs a robust extraction of these planes from which it deduces many useful information about the road and the obstacles located on its surface. Fig. 7 illustrates the outline of the process. From the two stereo images Fig. 7(a) and (b), a disparity map $I_{\Delta}$ Fig. 7(c) is computed (sum-ofsquare-differences (SSD) criteria are used to this purpose along edges). The disparity values are represented by a grey level according to the corresponding scale given on the left. Then, an accumulative projection of this disparity map is performed to build the "v-disparity" image $I_{v \Delta}$ Fig. 7(d). For the image line $i$, the abscissa $u_{M}$ of a point $M$ in $I_{v \Delta}$ corresponds to the disparity $\Delta_{M}$ and its grey level $i_{M}$ to the number of points with the same disparity $\Delta_{M}$ on the line $i: i_{M}=\sum_{P \in I_{\Delta}} \delta_{v_{P}, i} \delta_{\Delta_{P}, \Delta_{M}}$, where $\delta_{i, j}$ denotes the Kronecker delta.

From this "v-disparity" image, a robust extraction of straight lines is performed through a Hough transform. This extraction of straight lines Fig. 7(e) is equivalent to the extraction of the planes of interest taken into account in the modelization of the road scene.

3) Disparity-Map Improvement: In order to quickly compute the "v-disparity" image, a sparse and rough disparity map has been built. This disparity map may contain numerous false matches, which prevent us to use it as a depth map of the environment. Owing to the global surfaces extracted from the "v-disparity" image, false matches can be removed. In this aim, we check whether a pixel of the disparity map belongs to any global surface extracted using the same matching process. If this is the case, the same disparity value is mapped to the pixel and leads to Fig. 7(f). Details of this process can be found in [28]. Finally, this enhanced disparity map can be used as a depth map of the vehicle environment, since the depth $D$ of a pixel of disparity $\Delta$ is expressed by

$$
D=\frac{b\left(\alpha \cos \theta-\left(j-v_{\mathrm{o}}\right) \sin \theta\right)}{\Delta} .
$$

\section{Visibility-Distance Measurement}

\section{A. Direct Disparity-Contrast Combination}

Our first approach was to replace the computation of the horizontal local maxima of the gradient by the horizontal contrasts above $5 \%$. Therefore, the visibility distance is the distance to the pixel having the smallest disparity. This approach is 


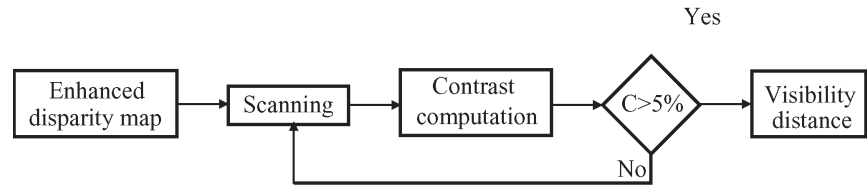

Fig. 8. Algorithm overview.

simple. Its main advantage is to replace the gradient threshold of the stereovision process, which is empirically chosen, by the contrast threshold of $5 \%$.

However, although the contrast computation time has been strongly reduced, it is still too large to be performed in real time on the whole image. Furthermore, it has to be performed on both images. We need $350 \mathrm{~ms}$ on a current-day PC to compute the horizontal contrasts on an image of resolution $380 \times 288$. By comparison, the computation time of horizontal gradient is less than $10 \mathrm{~ms}$, whatever the threshold.

\section{B. Fast Disparity-Contrast Combination}

The contrast computation locates precisely the edges, but is quite expensive in terms of computing times. Conversely, the gradient computation goes fast but spreads on the edges. Consequently, using the horizontal gradients, the "v-disparity" image is denser and faster to compute. The three-dimensional (3-D) surface extraction is also faster and more reliable. However, we must ensure that the gradient threshold is small enough to take most picture elements having a contrast above $5 \%$ into account, but large enough so as not to take noise into account. The noise measured on the cameras currently in use is Gaussian with a standard deviation $\sigma$ of one to two gray levels. Therefore, as not to take noise into account, the gradient threshold to consider is then $3 \sigma$, that is, to say six.

It is possible to draw advantage from both techniques while decreasing the computing time compared to the only use of horizontal contrasts. The method consists in computing the improved disparity map using the horizontal gradients higher than six and scanning it. Because most distant objects on the road surface are on the horizon line, the scanning starts from this location. Within each neighborhood where a point of disparity is known, the contrast is calculated. The process stops when a contrast above $5 \%$ is met. The visibility distance is then the depth of the picture element with a contrast above 5\%. The algorithm is summarized in Fig. 8 and now detailed in the following paragraph.

1) Developed Algorithm:

Some definitions:

1) Let $I_{d}$ denote the right image of the stereoscopic pair.

2) $V_{d}$ denotes the window belonging to $I_{d}$ centered on the pixel $(i, j)$.

3) $I_{\Delta}$ denotes the set of computed disparity.

4) $V_{\Delta}$ denotes the set of computed disparity belonging to $V_{d}$.

5) $I_{\mathrm{O}}$ denotes the pixels labeled as obstacles.

6) $V_{\mathrm{O}}$ denotes a window belonging to $I_{\mathrm{O}}$ centered on the pixel $(i, j)$.

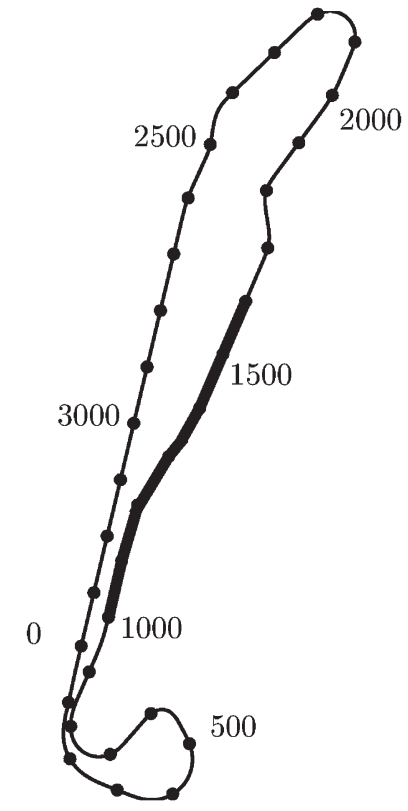

Fig. 9. Test track of Versailles Satory. The method is evaluated on the bold section of the figure between the coordinates 1000 and 1600 .

7) $\chi$ denotes the operator that returns the set of pixels belonging to $V_{d}$ with a contrast above $5 \%$.

8) $D$ denotes the operator that returns the depth in the road scene of a pixel $P(i, j)$ of disparity $\Delta$ [see (25)].

Scanning of the improved disparity map: Once the improved disparity map is achieved, we scan it with a sliding window $V_{d}$ from the top to the bottom and from the left to the right starting from the horizon line. Different situations may arise.

1) The considered window contains no pixels with a disparity value attached

$$
V_{\Delta}=\emptyset
$$

In this case, we pass to the next window location.

2) The considered window contains pixels with known disparity but also pixels labeled as obstacles

$$
V_{\Delta} \neq \emptyset \quad \text { and } \quad V_{\mathrm{o}} \neq \emptyset
$$

Because obstacle points can be closer to the sensor than points on the road surface, we pass to the next location.

3) The considered window contains pixels with a disparity value attached and no pixels labeled as obstacles

$$
V_{\Delta} \neq \emptyset \quad \text { and } \quad V_{\mathrm{o}}=\emptyset .
$$

In this case, we compute $\chi\left(V_{d}\right)$. If $\chi\left(V_{d}\right)=\emptyset$, we pass to the next window location. Otherwise, we can define the set $E_{v}$ of pixels with a disparity value attached having a contrast above 5\%

$$
E_{v}=V_{\Delta} \cap \chi\left(V_{d}\right) .
$$



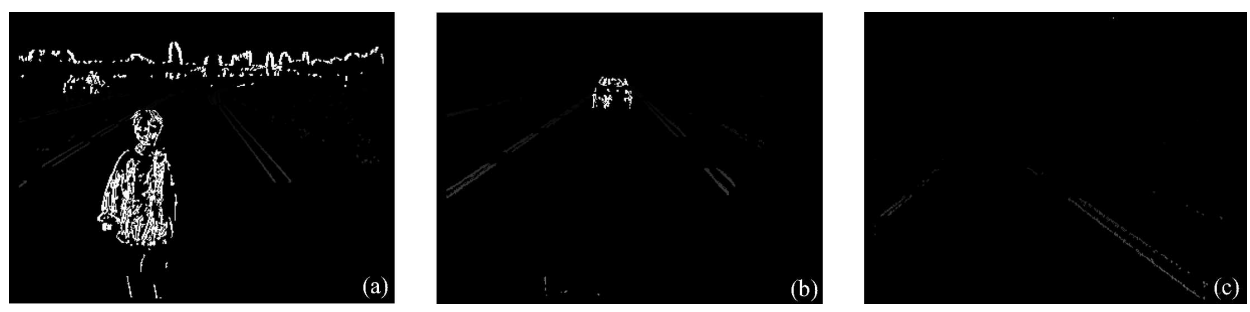

Fig. 10. Examples of disparity map of the vehicle environment (a) under sunny weather, (b) under foggy weather, and (c) under dense foggy weather before nightfall. White points are considered as obstacle points. The gray level of other points is proportional to their disparity.
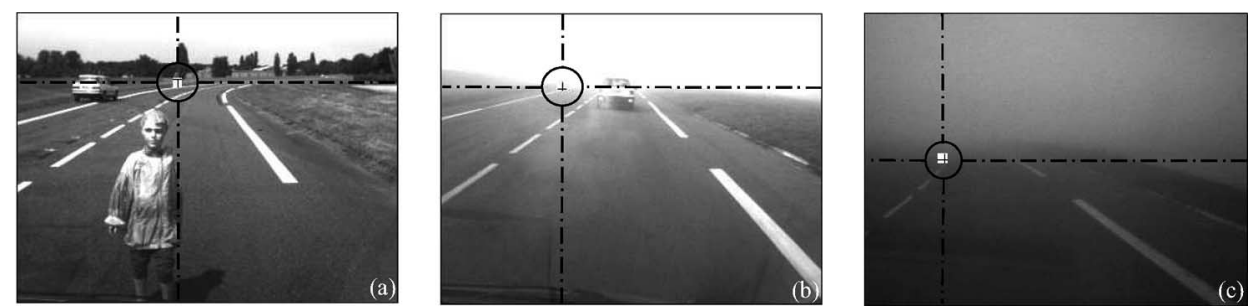

Fig. 11. Final result. The most distant window with a contrast above $5 \%$, in which a point of disparity is known, is painted white. The disparity point is represented with a black cross inside the white window. (a) Sunny weather $\left(V_{\mathrm{mob}} \approx 250 \mathrm{~m}\right)$, (b) foggy weather $\left(V_{\mathrm{mob}} \approx 75 \mathrm{~m}\right)$, and (c) dense foggy weather before nightfall $\left(V_{\mathrm{mob}} \approx 30 \mathrm{~m}\right)$.

Two subcases are thus considered.

1) If $E_{v}=\emptyset$, we pass to the next window location.

2) If $E_{v} \neq \emptyset$, the mobilized distance of visibility is the distance associated to the pixel belonging to $E_{v}$ with the smallest disparity, which means the greatest depth

$$
V_{\mathrm{mob}}=\max _{P \in E_{v}} D(P) .
$$

2) Potential Limits and Evolution: The described algorithm is well adapted to the structure of our disparity map, in which the disparity is computed only in pixels belonging to the road surface. However, in some cases, vertical objects can be very contrasted by day against the horizon sky and then better perceived than objects on the road surface. Consequently, in the future, it would be interesting to use a full 3-D disparity map. Unfortunately, in this case, the scanning mode of the image is not valid anymore. Consequently, we are also investigating sorting techniques to reduce the computational cost in such a case.

\section{Method Evaluation}

\section{A. Hardware Settings}

The whole process for building the depth map of the vehicle environment and computing the mobilized visibility distance by means of our Köhler's modified technique is performed within $60 \mathrm{~ms}$. The hardware used for the experiments is a Pentium IV $2.4 \mathrm{GHz}$. Images are grabbed using a Matrox Meteor II graphic card. The focal length is $8.5 \mathrm{~mm}$ and the image size is $380 \times$ 289. The program runs on the RT-Maps platform [29] and is compiled with the Intel C++ Compiler 8.0.

\section{B. Presentation of the Video Sequences Used}

This method has been tested on three video sequences, each of them containing around 1000 images. In the first sequence, the instrumented vehicle is following another car at various distances and stops in front of different obstacles like a pedestrian or a motorbike. The weather is sunny and clear. In the second sequence, the instrumented vehicle is following another car, which disappears progressively through the fog. On the last sequence, the vehicle is running on the track through a thick fog just before nightfall. All sequences were recorded on the same portion of our test-track facilities at Versailles Satory represented in Fig. 9. A sample of each sequence is given in Fig. 2.

\section{Results}

In Fig. 10, the results of the disparity map computation are presented. In Fig. 10(a), the pedestrian, the car, and the points beyond the horizon line are considered as obstacle points. The depth of the points on the road surface is computed. In the same way, in Fig. 10(b), the car is considered as an obstacle.

In Fig. 2, the results of local-contrast computation on the whole images are represented. In fact, as explained in Section IV-B, the contrast will not be computed on the whole image to save computing time. In Fig. 11, the final result is represented. The most distant window with a contrast above $5 \%$, in which a point of disparity is known, is painted white. The known disparity point is represented with a black cross inside this white window.

Finally, in Fig. 12, the curves of measured visibility distances are plotted for both video sequences. Under sunny weather, the maximum resolution of the stereoscopic sensor is reached. Under foggy weather, the measures are quite stable, which let 


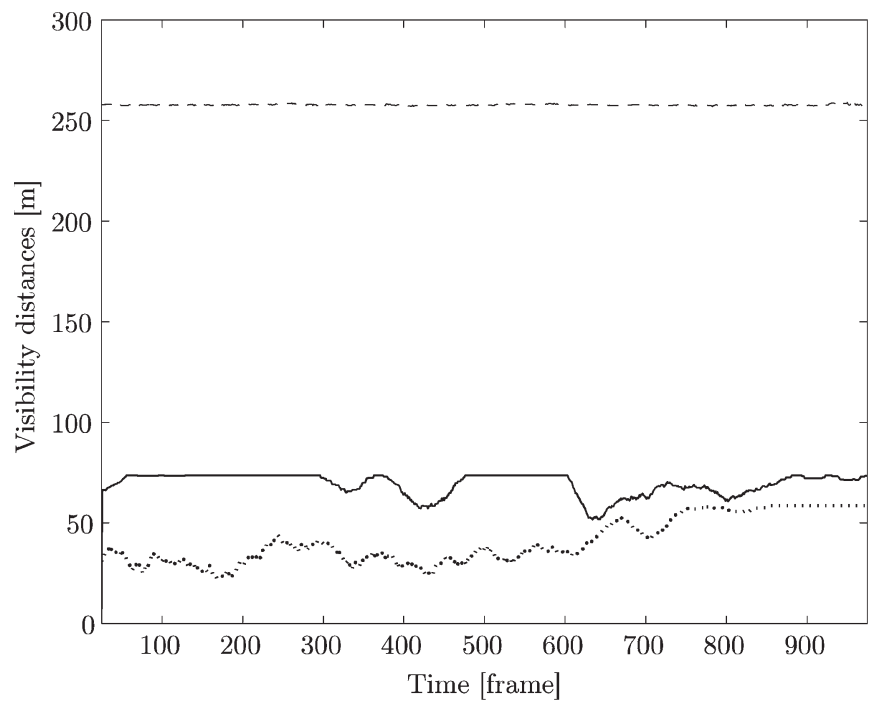

Fig. 12. Curves of measured mobilized visibility distances (- -) under sunny weather, (一) under foggy weather and (...) under dense foggy weather before nightfall.

us think that the method is efficient in adverse weather conditions. It is operative in every kind of meteorological condition. Because of its generic aspect and its good results, this method has been recently patented.

However, two hard points remain and need to be improved in the future. First, we must study the adequation of the threshold of 5\%, proposed by the CIE, in different meteorological and illumination conditions. An adaptive threshold depending on the surrounding luminance could be more adapted. Second, the use of sparse disparity maps is not always adequate in our case, where the objects are very low contrasted. Indeed, the disparity is then known on very few pixels. We think that quasi-dense matching techniques should improve the method.

\section{CONCLUSION}

In this paper, we presented a generic method to estimate the mobilized visibility distance, which is the distance of the most distant picture element on the road surface with a contrast above 5\%. This concept is close to the meteorological visibility distance. We use the "v-disparity" stereovision approach to build a depth map of the vehicle environment. We combine this map with the computation of local contrasts by means of a technique inspired by Köhler. The whole process is performed in real time. This technique, which has been recently patented, has very few assumptions. Consequently, it is operative under every meteorological condition and is stable in adverse weather conditions.

\section{REFERENCES}

[1] V. Cavallo, M. Colomb, and J. Dore, "Distance perception of vehicle rear lights in fog," Hum. Factors, vol. 43, no. 3, pp. 442-451, 2001.

[2] E. Dumont and V. Cavallo, "Extended photometric model of fog effects on road vision," Transp. Res. Rec.: J. Transp. Res. Board, no. 1862, pp. 77-81, 2004.

[3] N. Hautière and D. Aubert, "Driving assistance: Automatic fog detection and measure of the visibility distance," in Proc. ITS World Congr., Madrid, Spain, Nov. 2003
[4] C. Bush and E. Debes, "Wavelet transform for analyzing fog visibility," IEEE Intell. Syst., vol. 13, no. 6, pp. 66-71, Nov./Dec. 1998

[5] D. Pomerleau, "Visibility estimation from a moving vehicle using the ralph vision system," in Proc. IEEE Conf. Intell. Transp. Syst., Nov. 1997, pp. 906-911.

[6] G. Yahiaoui and P. Da Silva Dias, "In board visibility evaluation for car safety applications: A human vision modelling based approach," in Proc. ITS World Congr., Madrid, Spain, Nov. 2003.

[7] S. Winkler, "Issues in vision modeling for perceptual video quality assessment," Signal Process., vol. 78, no. 2, pp. 231-252, Oct. 1999.

[8] J. Tang, E. Peli, and S. Acton, "Image enhancement using a contrast measure in the compressed domain," IEEE Signal Process. Lett., vol. 10, no. 10, pp. 289-292, Oct. 2003.

[9] M. Jourlin and J.-C. Pinoli, "Logarithmic image processing," in Advances in Imaging and Electron Physics, vol. 115. New York: Academic, 2001, pp. 129-196.

[10] R. Sivaramakrishna, N. A. Obuchowski, W. A. Chilcote, G. Cardenosa, and K. A. Powell, "Comparing the performance of mammographic enhancement algorithms: A preference study," Amer. J. Roentgenol., vol. 175 , no. 1, pp. 45-51, 2000.

[11] J. P. Oakley and B. L. Satherley, "Improving image quality in poor visibility conditions using a physical model for contrast degradation," IEEE Trans. Image Process., vol. 7, no. 2, pp. 167-179, Feb. 1998.

[12] S. G. Narasimhan and S. K. Nayar, "Contrast restoration of weather degraded images," IEEE Trans. Pattern Anal. Mach. Intell., vol. 25, no. 6, pp. 713-724, Jun. 2003.

[13] F. Cozman and E. Krotkov, "Depth from scattering," in Proc. IEEE Conf. Comput. Vis. Pattern Recog., Jun. 1997, pp. 801-806.

[14] Y. Y. Schechner, S. G. Narasimhan, and S. K. Nayar, "Instant dehazing of images using polarization," in Proc. IEEE Conf. Comput. Vis. Pattern Recog., Dec. 2001, pp. I-325-I-332.

[15] M. Cheriet, J. N. Said, and C. Y. Suen, "A recursive thresholding technique for image segmentation," IEEE Trans. Image Process., vol. 7, no. 6, pp. 918-921, Jun. 1998.

[16] Y. He, H. Wang, and B. Zhang, "Color-based road detection in urban traffic scenes," IEEE Trans. Intell. Transp. Syst., vol. 5, no. 4, pp. 309-318, Dec. 2004.

[17] R. Aufrère, R. Chapuis, and F. Chausse, "A fast and robust vision algorithm to locate a vehicle on a non-structured road," Int. J. Rob. Res., vol. 19, no. 5, pp. 411-423, May 2000.

[18] M. Bertozzi, A. Broggi, M. Cellario, A. Fascioli, and M. Porta, "Artificial vision in road vehicles," Proc. IEEE-Special Issue on Technology and Tools for Visual Perception, vol. 90, no. 7, pp. 1258-1271, Jul. 2002.

[19] S.-S. Ieng, J.-P. Tarel, and P. Charbonnier, "Robust estimation for camera based detection and tracking," Traitement du Signal, vol. 21, no. 3, pp. 205-226, 2004.

[20] R. Chapuis, R. Aufrère, and F. Chausse, "Accurate road following and reconstruction by computer vision," IEEE Trans. Intell. Transp. Syst., vol. 3, no. 4, pp. 261-270, Dec. 2002.

[21] U. Franke and C. Rabe, "Kalman filter based depth from motion with fast convergence," in Proc. IEEE Intell. Vehicles Symp., Las Vegas, NV, 2005, pp. $180-185$.

[22] M. Betke and H. Nguyen, "Highway scene analysis from a moving vehicle under reduced visibility conditions," in Proc. IEEE Int. Conf. Intell. Vehicles, Oct. 1998, vol. 1, pp. 131-136.

[23] A. Broggi, A. Fascioli, C. G. L. Bianco, and A. Piazzi, "Visual perception of obstacles and vehicles for platooning," IEEE Trans. Intell. Transp. Syst., vol. 1, no. 3, pp. 164-176, Sep. 2000.

[24] F. Deschênes and D. Ziou, "Depth from defocus estimation in spatial domain," Comput. Vis. Image Underst., vol. 81, no. 2, pp. 143-165, Feb. 2001.

[25] R. Zhang, P.-S. Tsai, J. E. Cryer, and M. Shah, "Shape from shading: A survey," IEEE Trans. Pattern Anal. Mach. Intell., vol. 21, no. 8, pp. 690-706, Aug. 1999.

[26] O. Faugeras and Q.-T. Luong, The Geometry of Multiple Images. Cambridge, MA: MIT Press, 2001.

[27] R. Labayrade, D. Aubert, and J.-P. Tarel, "Real time obstacle detection in stereovision on non flat road geometry through v-disparity representation," in Proc. IEEE Intell. Vehicles Symp., 2002, pp. 646-651.

[28] R. Labayrade and D. Aubert, "In-vehicle obstacles detection and characterization by stereovision," in Proc. 1st Int. Workshop In-Vehicle Cognitive Comput. Vis. Syst., Graz, Austria, Nov. 2003, pp. 13-19.

[29] F. Nashashibi, B. Steux, P. Coulombeau, and C. Laurgeau, "Rt- maps a framework for prototyping automotive multi-sensor applications," in Proc. IEEE Intell. Vehicles Symp., Dearborn, MI, Oct. 2000, pp. 99-103. 


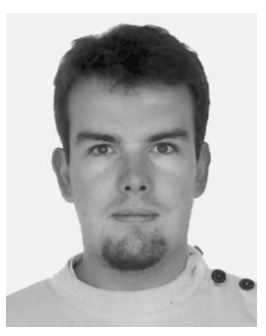

Nicolas Hautière received the M.S. degree in civil engineering from the National School of State Public Works (ENTPE) Engineer School, Lyon, France, in 2002 and the M.S. and Ph.D. degrees in computer vision from the University Jean Monnet, SaintEtienne, France, in 2002 and 2005, respectively.

Since 2006, he has been a Researcher at LCPC. His research interests span computer vision, robotics, biologically inspired vision, and advanced driving assistance systems. He has published several papers on in-vehicle estimation of meteorological visibility distance.

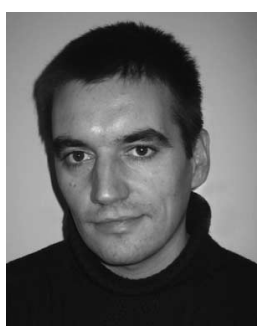

Raphaël Labayrade was born in France in 1976. $\mathrm{He}$ received the M.S. degree in civil engineering from the University of Saint-Etienne, Saint-Etienne, France, and a degree from the National School of State Public Works (ENTPE) engineer school, Lyon, France, both in 2000 , and the Ph.D. degree in computer science from the University of Paris 6, Jussieu, France, in 2004.

He has been a Researcher at French National Institute For Transport and Safety Research (INRETS) since 2004 in the perception team of the VehiclesInfrastructure-Drivers Interactions Research Laboratory (LIVIC) Department and works on automated highway and on on-board driving assistance systems. His main work deals with obstacle detection using stereovision, but he is also interested in road-lane recognition and data fusion. He is involved in perception tasks in various European and French projects dealing with intelligent vehicles (Prevensor, DO30, CVIS). He teaches at Jussieu (Paris VI), Ecole Nationale des Ponts et Chaussées, University of Versailles, Versailles, France, and in private companies. He is the author and coauthor of several technical papers.

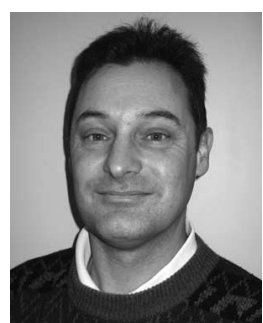

Didier Aubert received the M.S. and Ph.D. degrees in computer science from the National Polytechnical Institute of Grenoble (INPG), Grenoble, France, in 1985 and 1989 , respectively.

From 1989 to 1990, he was a Research Scientist on the development of an automatic-road following system for the Navigatory Laboratory (NAVLAB) at Carnegie Mellon University, Pittsburgh, PA. From 1990 to 1994, he worked in the research department of a private company Industrie et Technologie de la Machine Intelligente (ITMI). During this period, he was a Project Leader of several projects dealing with computer vision. $\mathrm{He}$ has been a Researcher at French National Institute For Transport and Safety Research (INRETS) since 1995 and has been working on road-traffic measurements, crowd monitoring, automated highway systems, and driving assistance systems for vehicles. He is an image processing expert for several companies, teaches at Universities (Paris VI, Paris XI, Ecole Nationale des Ponts et Chaussées (ENPC), Ecole Nationale Supérieure des Télécommunications (ENST), Ecole Nationale Supérieure des Mines de Paris (ENSMP), and Evry University) and is on the Editorial Board of Research-TransportSafety (RTS). 